\title{
DIRAC PARTICLES IN COULOMB LIKE FIELD IN FLRW-SPACE
}

\author{
S. K. Sharma*, P. R. Dhungel**, and U. Khanal*** \\ *B.P. Koirala Memorial Planetarium, Observatory and Science Museum Development Board, MoEST, Kathmandu, \\ Nepal. \\ **Department of Physics, St. Xavier's College, Tribhuvan University, Kathmandu, Nepal. \\ *** Ratobangala School, Lalitpur, Nepal.
}

\begin{abstract}
Behaviour of the Dirac particle in Coulomb like field in FLRW space is investigated. Firstly, the Maxwell equations, in terms of the vector potentials are solved to identify the Lorentz and Coulomb like gauges. The radial Coulomb like potential is solved in terms of Legendre functions. Then the Dirac equation is generalized to include this potential and the angular part is separated and solved. The radial and temporal parts of the massless case is also separated and solved. But the massive case remains coupled. This is still reduced to the case where the Dirac particle can be represented as being in a combined gravitational and electric potential. This effective potential is found to develop an attractive well, which may require a revisit to the recombination era.
\end{abstract}

Key words: FLRW spacetime; Dirac field; Coulomb like potential; NP formalism.

\section{INTRODUCTION}

All matter being ultimately fermions, study of Dirac field is very important to understand the nature and behaviour of the constituents of the universe and its evolutions. Such study becomes much more convenient to formulate in the

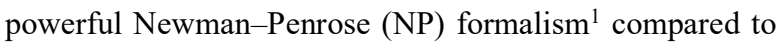
the conventional tensor method ${ }^{2,3}$. Although NP formalism was primarily used to study quantum fields in black hole space times, we extended its application to write and solve Maxwell, Dirac ${ }^{4,5}$ and gravitational perturbation equations ${ }^{6}$ in FLRW space-time.

In this work, we use the same formalism and notations as in Chandrasekhar $^{7}$ to study the behaviour of the Dirac particles in Coulomb like gauge field in FLRW spacetime. In the first part of the next section, we set up the preliminaries to write the Maxwell's equations in terms of the gauge potentials to identify the Lorentz and Coulomb like gauges, and solve for the potential due to a point charge. In the remaining part of that section, the Dirac equa- tions have been solved to obtain the Coulomb like potenti als and force fields. In the third section, some discussions along with concluding remarks have been made.

\section{LORENTZ LIKE GAUGE AND DIRAC FIELD IN COULOMB LIKE POTENTIAL}

\section{Lorentz like gauge}

In our chosen tetrad frame ${ }^{1,4,7}$ for the FLRW spacetime $l_{\mu}=$ $[1,-1,0,0], \quad n_{\mu}=\frac{a^{2}}{2}[1,1,0,0], \quad m_{\mu}=$ $-\frac{a s}{\sqrt{2}}[0,0,1, i \sin \theta]$ and the complex conjugate $\bar{m}_{\mu}$, the electromagnetic 4-vector potentials are given by $A_{l}=$ $A_{\mu} l^{\mu}=\frac{1}{a^{2}}\left(A_{\eta}+A_{r}\right), \quad A_{n}=\frac{1}{2}\left(A_{\eta}-A_{r}\right), \quad A_{m}=$ $\frac{1}{\sqrt{2} a S}\left(A_{\theta}+\frac{i}{\sin \theta} A_{\phi}\right)$ and $A_{\bar{m}}$, where $S=\frac{\sqrt{k} r}{\sqrt{k}}=\left\{\begin{array}{cl}\sin r, & k=1 \text { for closed } \\ r, & k=0 \text { for flat } \\ \sinh r, & k=-1 \text { for open }\end{array}\right.$

The Maxwell scalar representing the field strengths are then,

$$
F_{\mu \nu} l^{\mu} m^{v}=\varphi_{0}=\frac{1}{a^{3} S} \mathcal{D}^{-} a S A_{m}+\frac{1}{\sqrt{2} a S} \mathcal{L}_{0}^{-} A_{l}
$$

Author for correspondence: P. R. Dhungel, Department of Physics, St. Xavier's College, Tribhuvan University, Kathmandu, Nepal.

Email: premrd@hotmail.com

Received: 7 Nov 2020; First Review: 19 dec 2020; Second Review: 24 dec 2020; Accepted: 26 Dec 2020. 


$$
\begin{aligned}
& \frac{1}{2} F_{\mu \nu}\left(l^{\mu} n^{v}+m^{\mu} \bar{m}^{v}\right)=\varphi_{1} \\
& =-\frac{1}{2 a}\left(\mathcal{D}^{+} a^{2} \frac{A_{l}}{2}+\mathcal{D}^{-} A_{n}\right)+\frac{1}{2 \sqrt{2} a S}\left(\mathcal{L}_{1}^{-} A_{\bar{m}}-\mathcal{L}_{1}^{+} A_{m}\right) .
\end{aligned}
$$

and,

$F_{\mu \nu} \bar{m}^{\mu} n^{\nu}=\varphi_{2}=\frac{1}{2 a S} \mathcal{D}^{+} a S A_{\bar{m}}-\frac{1}{\sqrt{2} a S} \mathcal{L}_{0}^{-} A_{n} \ldots($

We will be using the operator $\mathcal{D}_{n}^{ \pm}=\left(\frac{\partial}{\partial r} \mp \frac{\partial}{\partial \eta}+n \frac{S \prime}{S}\right)$ and $\mathcal{L}_{n}^{ \pm}=\left(\frac{\partial}{\partial \theta} \mp \frac{1}{\sin \theta} \frac{\partial}{\partial \varphi}+n \cot \theta\right)$. The number of factors of $l^{\mu}$ minus those of $n^{\mu}$ is the boost weight and that of $m^{\mu}$ minus $\bar{m}^{\mu}$ is spin weight. So, $\varphi_{0}$ is a field of helicity $+1, \varphi_{2}$ of 1 and $\varphi_{1}$ of zero. Substituting these expressions for $\varphi^{\prime}$ s in the Maxwell equations leads to

$$
\begin{aligned}
& \mathcal{D}^{-}\left[S^{2}\left(\mathcal{D}^{+} \frac{a^{2}}{2} A_{l}+\mathcal{D}^{-} A_{n}\right)-\frac{a S}{\sqrt{2}}\left(\mathcal{L}_{1}^{-} A_{\bar{m}}+\mathcal{L}_{1}^{+} A_{m}\right)\right] \\
& +2 \mathcal{L}_{1}^{+} \mathcal{L}_{0}^{-} \frac{a^{2}}{2} A_{l}=-a^{4} S^{2} J_{l}
\end{aligned}
$$

$\mathcal{D}^{+}\left[S^{2}\left(\mathcal{D}^{+} \frac{a^{2}}{2} A_{l}+\mathcal{D}^{-} A_{n}\right)+\frac{a S}{\sqrt{2}}\left(\mathcal{L}_{1}^{-} A_{\bar{m}}+\mathcal{L}_{1}^{+} A_{m}\right)\right]$

$+2 \mathcal{L}_{1}^{-} \mathcal{L}_{0}^{-} A_{n}=-2 a^{2} S^{2} J_{n}$

$$
\begin{aligned}
& S^{2} \mathcal{D}^{ \pm} \mathcal{D}^{\mp} a S\left(\begin{array}{c}
A_{m} \\
A_{\bar{m}}
\end{array}\right)+ \\
& \frac{\mathcal{L}_{0}^{\mp}}{\sqrt{2}}\left[S^{2}\left(\mathcal{D}^{-} A_{n}-\mathcal{D}^{+} \frac{a^{2}}{2} A_{l}\right) \mp \frac{a S}{\sqrt{2}}\left(\mathcal{L}_{1}^{-} A_{\bar{m}}-\mathcal{L}_{1}^{+} A_{m}\right)\right] \\
& =-a^{3} S^{3}\left(\begin{array}{l}
J_{m} \\
J_{\bar{m}}
\end{array}\right)
\end{aligned}
$$

A Lorentz like gauge can immediately be identified as

$S^{2}\left(-\mathcal{D}^{-} A_{n}+\mathcal{D}^{+} \frac{a^{2}}{2} A_{l}\right)+\frac{a S}{\sqrt{2}}\left(\mathcal{L}_{1}^{-} A_{\bar{m}}+\mathcal{L}_{1}^{+} A_{m}\right)=0$

Whence the Maxwell Eqs. (5a, b, c) become

$$
\left[\mathcal{D}^{ \pm} S^{2} \mathcal{D}^{\mp}+\mathcal{L}_{0}^{\mp} \mathcal{L}_{1}^{ \pm}\right]\left(\begin{array}{c}
a^{2} A_{l} \\
A_{n}
\end{array}\right)=-a^{2} S^{2}\left(\begin{array}{l}
J_{l} \\
J_{n}
\end{array}\right)
$$

and

$$
\left[S^{2} \mathcal{D}^{\mp} \mathcal{D}^{ \pm}+\mathcal{L}_{0}^{\mp} \mathcal{L}_{1}^{ \pm}\right] a S\left(\begin{array}{c}
A_{m} \\
A_{\bar{m}}
\end{array}\right)=-a^{3} S^{3}\left(\begin{array}{c}
J_{m} \\
J_{\bar{m}}
\end{array}\right)
$$

We call (6) Lorentz like condition. It is the vanishing of the 4-divergence of $\frac{A^{\mu}}{(\mathrm{aS})^{2}}$ rather than that of $A^{\mu}$.

We have already used the s-spin weighted spherical harmonics ${ }_{s} Y_{l}^{m}$ that obey the spin lowering (raising) operations $\mathcal{L}_{S}^{ \pm}{ }_{ \pm s} Y_{l}^{m}= \pm L_{s-1} \pm(s-1) Y_{l}^{m}$ where $L_{s}^{2}=(l-$ $s)(l+s+1)$ and the s-boost weight functions ${ }_{s} Z_{k}^{\omega}$ obeying $S \mathcal{D}_{-s \pm s}^{ \pm} Z_{k}^{\omega}= \pm i K_{s-1} \pm(s-1) Z_{k}^{\omega}$ where $K_{s}^{2}=(k-$ $s)(k+s+1)$.

We also identified ${ }_{p} Y_{l}^{m}=\frac{N_{1}}{\sqrt{2 \pi}} e^{i m \varphi}(1-\cos \theta)^{\frac{m+p}{2}}(1+$ $\cos \theta)^{\frac{m-p}{2}} P_{l-m}^{(m+p, m-p)}(\cos \theta)$ as spherical harmonics formed with Jacobi polynomials ${ }^{8} P_{n}^{(\alpha, \beta)}$, and ${ }_{p} Z_{k}^{\omega}=$ $\frac{N_{2}}{\sqrt{2 \pi}} e^{-i m \eta}(1-i \cot r)^{-\frac{\omega+p}{2}}(1+$

$i \cot r)^{-\frac{\omega-p}{2}} P_{\omega-k-1}^{(-\omega-p,-\omega+p)}(i \cot r)$. So, we can just read off the solutions of (7) as

$\left(\begin{array}{c}a^{2} A_{l} \\ A_{n}\end{array}\right) \sim \frac{1}{S} \pm 1 Z_{k} Z_{0} Y_{l}^{m}$ and $a S\left(\begin{array}{c}A_{m} \\ A_{\bar{m}}\end{array}\right) \sim{ }_{0} Z_{k}^{\omega}{ }_{11} Y_{l}^{m}$.

For solutions of homogenous equations it is just the condition $k=l$.

For Coulomb like gauge we add the equations (5a) and (5b) and find that the condition

$\frac{\partial}{\partial r} S^{2}\left(\frac{a^{2}}{2} A_{l}-A_{n}\right)+\frac{a S}{\sqrt{2}}\left(\mathcal{L}_{1}^{-} A_{\bar{m}}+\mathcal{L}_{1}^{+} A_{m}\right)=0$

gives

$\left[\frac{\partial}{\partial r} S^{2} \frac{\partial}{\partial r}+\mathcal{L}_{1}^{+} \mathcal{L}_{0}^{-}\right] A_{\eta}=-n a^{3} S^{2}=-n_{0} a_{0}^{3} S^{2}$.

The potential of a point charge q located at the origin is found to be

$$
A_{\eta}=\frac{q}{4 \pi} \frac{S^{\prime}}{S}==\frac{q}{4 \pi} \begin{cases}\cot r, & k=1 \\ \frac{1}{r}, & k=0 \\ \operatorname{coth} r, & k=-1\end{cases}
$$

We may proceed to solve the general equation (9) for the Green's function by standard techniques ${ }^{9}$ to find (for the closed Universe with $k=1$ )

$G\left(r, r^{\prime}\right)=$

$\frac{\pi}{2} \frac{1}{\sqrt{\sin r \sin r^{\prime}}} \sum_{l=0}^{\infty} \frac{(-l)(2 l+1)}{4 \pi} P_{\frac{1}{2}}^{-\left(l+\frac{1}{2}\right)}\left(\cos r^{\prime}\right) P_{\frac{1}{2}}^{\left(l+\frac{1}{2}\right)}(\cos r) P_{l} \cos \beta$ 
for $r>r^{\prime}$ and $r r^{\prime}$ for $r<r^{\prime}$. We expect that an addition formula for Associated Legendre functions $P_{\frac{1}{2}}^{ \pm\left(l+\frac{1}{2}\right)}(\cos r)$ give

$G\left(\boldsymbol{r}, \boldsymbol{r}^{\prime}\right)=-\frac{1}{4 \pi} \cot \rho$

where $\cos \rho=\cos r \cos r^{\prime}+\sin r \sin r^{\prime} \cos \beta$

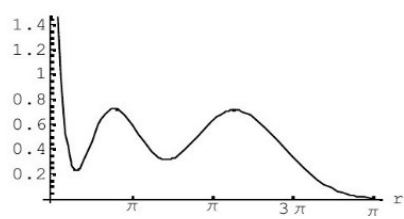

(a)

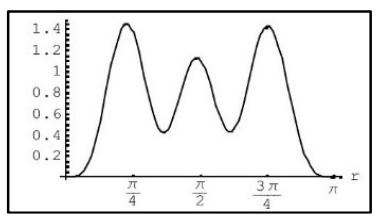

(b)

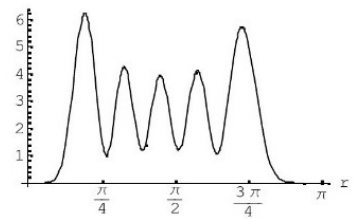

(c)

Figure 1: Plot of $\left|R_{+}\right|^{2}+\left|R_{-}\right|^{2}$ representing the relative probability against $r$ for $\alpha z=1$. (a) for $n=2 \& l=1 / 2$, (b) for $n=2 \& l=3 / 2$, (c) for $n=4 \& l=9 / 2$.

\section{Dirac field in a Coulomb-like potential}

Next we use the previous result to look at the analogue of the hydrogen like atom in an expanding universe. We have dealt with the Dirac equation in another work ${ }^{4}$ and the radial part is given by the equation

$\mathcal{D}^{ \pm} \Phi_{ \pm}=-\left(\frac{\lambda}{s} \pm i M a\right) \Phi_{\mp}$

where $\lambda=l+\frac{1}{2}$ and $l=\frac{1}{2}, \frac{3}{2}, \frac{5}{2}, \ldots \ldots \ldots$

We have just changed the sign of the eigenvalue $\lambda$ of eigenfunction $\mathcal{L}_{\frac{1}{2}}^{ \pm} \pm \frac{1}{2} Y_{l}^{m}= \pm\left(l+\frac{1}{2}\right)_{{ }_{1} \frac{1}{2}} Y_{l}^{m}$ to be consistent with other ${ }_{s} Y_{l}^{m}$.
We just make the minimal substitution, $\partial_{\mu} \rightarrow \partial_{\mu}+i e A_{\mu}$. With only a point charge $z e$ at the origin and a Dirac field of negative charge (-e), we have

$\left[\frac{\partial}{\partial r} \mp\left(\frac{\partial}{\partial \eta}-i z \alpha \frac{S \prime}{S}\right)\right] \Phi_{ \pm}=-\left(\frac{\lambda}{s} \pm i M a\right) \Phi_{\mp}$

where $\alpha=\frac{e^{2}}{\hbar c}$ is the fine structure constant and the minus sign is to represent attractive potential. The massless case $\mathrm{M}=0$ is easily solved with time dependence $e^{-i \omega \eta}$ to give the radial part as

$R_{ \pm}(r) \sim(1-i \cot r)^{-\left(b-\frac{1}{2}\right)}(1+i \cot r)^{-\left(c-\frac{1}{2}\right)} P_{n}^{(-b,-c)}(i \cot r) \ldots$

Where $\omega=n+\frac{1}{2}+\sqrt{\left(l+\frac{1}{2}\right)^{2}-\alpha^{2} z^{2}}, b=\omega-i \alpha z \pm \frac{1}{2}$,

and $c=\omega+i \alpha z \mp \frac{1}{2}$.

Plot of $\left|R_{+}\right|^{2}+\left|R_{-}\right|^{2}$ representing the relative probability, for some typical values of az, 1 and $n$ are shown in Fig. (1).

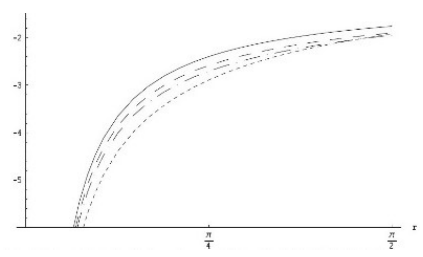

(a)

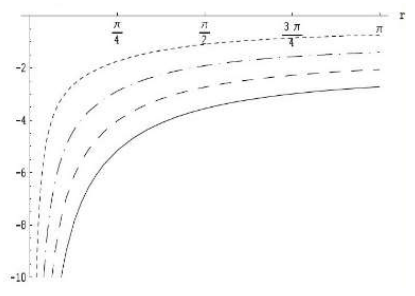

(b)

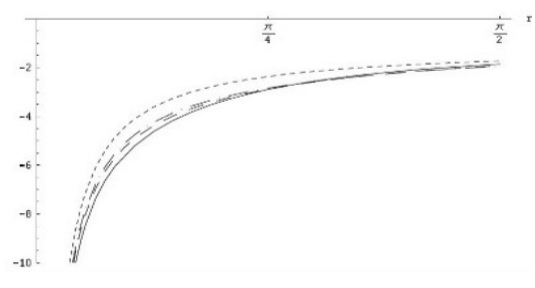

(c)

Figure 2: The modified vector potentials $A_{\eta}$ with various parameters varied. (a) $k=0, a=1, \alpha z=1$ with $l$ varied. Dotted for $l=1 / 2$, dot-dashed for $l=3 / 2$, dashed for $l=5 / 2$ and solid for $l=15 / 2$. (b) $k=0, l=1 / 2$ with $\alpha z$ varied. Dotted for $\alpha z=0.5$, dot-dashed for $\alpha z=1$, dashed for $\alpha z=1.5$ and solid for $\alpha z=2$. (c) $k=1, l=1 / 2, \alpha z=1$ with $a$ varied. Dotted for $a=0.1$, dotdashed for $a=0.8$, dashed for $a=1$ and solid for $a=1.5$. 
For the massive case, although the equations can be decoupled for $\varphi_{+}$and $\varphi_{-}$, we are unable to separate the variables $r$ and $\eta$. We can get an insight into the behaviour by substituting $\frac{\lambda}{S} \pm i M a=\chi e^{ \pm i \xi}$, where $\chi^{2}=\frac{\lambda^{2}}{S^{2}}+M^{2} a^{2}$ and $\tan \xi=\frac{M a S}{\lambda}$, and $\psi_{ \pm}=e^{\mp \frac{i \xi}{2}} \varphi$ to give

$\left[\left(\frac{\partial}{\partial r}-\frac{i}{2} \frac{\partial \xi}{\partial \eta}\right) \mp\left(\frac{\partial}{\partial \eta}-i z \alpha \frac{S^{\prime}}{S}-\frac{i}{2} \frac{\partial \xi}{\partial r}\right)\right] \Psi_{ \pm}=-\chi \Psi_{\mp} \ldots$

We can think of derivatives of $\xi$ as adding on to the vector potentials as gravitational counter parts. In particular, the attractive modified potentials are

$A_{r}=-\frac{1}{2} \frac{\partial \xi}{\partial \eta}=-\frac{1}{2} \sin \xi \cos \xi \frac{1}{a} \frac{\partial a}{\partial \eta}$

$A_{\eta}=-\alpha z \frac{S^{\prime}}{S}-\frac{1}{2} \frac{\partial \xi}{\partial r}-\alpha z \frac{S^{\prime}}{S} r-\frac{1}{2} \sin \xi \cos \xi \frac{S \prime}{S}$

These potentials are plotted in Fig. (2) and (3).
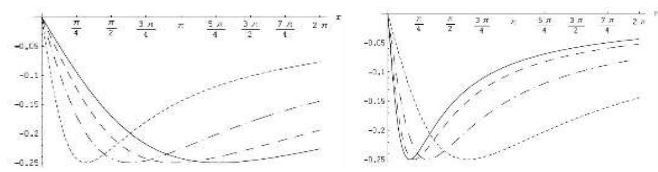

(a)

(b)
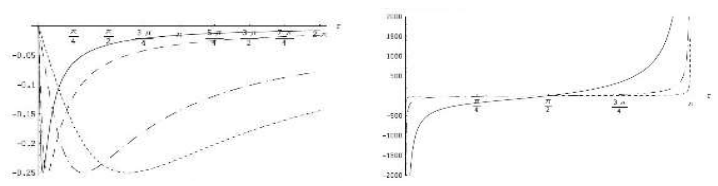

(c)

(d)

Figure 3: The modified vector potentials $A_{r}$ with various parameters varied. (a) $k=0, a=1$, with $l$ varied. Dotted for $l=1 / 2$, dot-dashed for $l=3 / 2$, dashed for $l=5 / 2$ and solid for $l=7 / 2$. (b) $k=0, l=1 / 2$ with $a$ varied. Dotted for $a=0.5$, dot-dashed for $a=1$, dashed for $a=1.5$ and solid for $a=1$. 8. (c) $k=0, l=1 / 2, a=1$ with $m$ varied. Dotted for $m=0.5$, dot-dashed for $m=1$, dashed for $m=5$ and solid for $m=10$. (d) $k=1, l=1 / 2, a=1$ with $\alpha z$ varied. Dotted for $\alpha z=1$, dot-dashed for $\alpha z=10$ and solid for $\alpha z=100$.

The force field due to these potentials is

$$
\begin{aligned}
F_{\eta r} & =\frac{\partial A_{r}}{\partial \eta}-\frac{\partial A_{\eta}}{\partial r} \\
& =-\frac{\alpha z}{S^{2}}-\frac{1}{2} \sin \xi \cos \xi\left[\frac{a^{\prime \prime}}{a}+K-2 \sin ^{2} \xi\left(\left(\frac{a^{\prime}}{a}\right)^{2}+K-\frac{1}{S^{2}}\right)\right]
\end{aligned}
$$

$$
\begin{aligned}
=-\frac{\alpha z}{S^{2}}-\frac{1}{2} \sin \xi & \cos \xi\left[\frac{4 \pi G}{3} a^{2}(\rho-3 P)\right. \\
& \left.-2 \sin ^{2} \xi\left(\frac{8 \pi G}{3} a^{2} \rho-\frac{1}{S^{2}}\right)\right]
\end{aligned}
$$

This force field is also shown in Fig. (4) at different phases of expansion and various conditions.

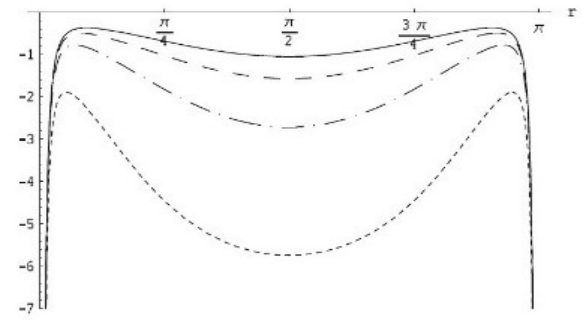

(a)

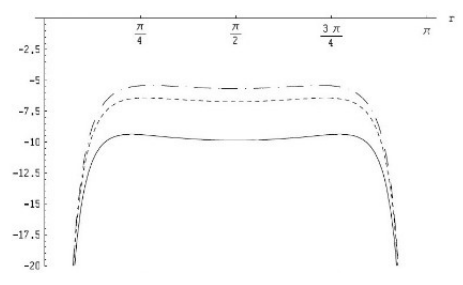

(b)

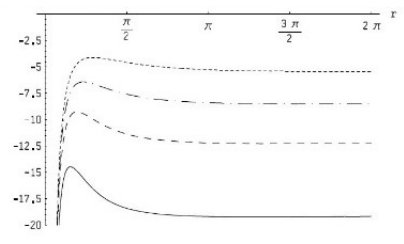

(c)

Figure 4: The force field $F_{\eta r}$ plotted against $r$. (a) matter-dominated closed $(K=1)$ universe, $a=1, \alpha z=.01$. Dotted for $l=1 / 2$, dot-dashed for $l=3 / 2$, dashed for $l=5 / 2$ and solid for $l=7 / 2$, (b) closed (K=1) universe, $l=1 / 2, a=1, \alpha z=1$. Dotted for matter-dominated, dotdashed for radiation- dominated, and solid for vacuum dominated, (c) matter- dominated, and solid for vacuum dominated, (c) matterdominated flat $(K=0)$ universe, $l=1 / 2, \alpha z=1$. Dotted for $a=0.8$, dot-dashed for $a=1$, dashed for $a=1.2$ and solid for $a=1.5$.

\section{CONCLUSION AND DISCUSSION}

In this work we have tried to investigate the behaviour of the Dirac particle in the FLRW spacetime endowed with Maxwell field. To do this, we first derived a representation for the Maxwellian vector potential that is analogous to the Lorentz and Coulomb gauges. Lorentz like gauge is quite evident, just requiring the vanishing of the four divergence of $\frac{A^{\mu}}{(a S)^{2}}$. The solution for the potentials become immediate 
in terms of the appropriate combinations of boost and spin weighted functions.

We also identify a Coulomb like gauge in which the potential reduces to $1 / r$ in flat space, and solve for the appropriate Green function.

Then we use this condition to write the Dirac equation for an electron in the Coulomb like field of a point positive charge. We make the minimal substitution of the potential in the covariant derivative. The massless case can be completely solved, and the energy levels exhibit a quantized structure [Eq. 14]. But, we are not able to separate the radial and temporal parts of the equations for massive electron. Nonetheless, we are able to reduce the equations to where the scale factor and curvature of the FLRW spacetime appear as a gravitational counterpart, adding on to the Coulomb potential. Fig. 3 shows that this modified potential always develops a minimum, allowing bound states. Further work is necessary to determine whether this consequence will lead to a re-evaluation of the recombination era of the Universe.

\section{REFERENCES}

1. Newman E.T. and Penrose, R. 1962. An Approach to Gravitational Radiation by a Method of Spin Coefficients. J. Math. Phys. 3: 566.

2. Misner, C.W., Thorne, K.S. and Wheeler, J.A. 1972. Gravitation, W. H. Freeman and Company, San Francisco.
3. Weinberg, S. 1972. Gravitation and Cosmology. John Wiley and Sons, Inc., New York.

4. Dhungel, P.R. and Khanal, U. 2013. Dirac Field in FRW Spacetime: Current and Energy Momentum, Chinese Journal of Physics. 51(5): 882.

5. Khanal, U. 2006. Electrodynamics in FRW Universe: Maxwell and Dirac fields in NP formalism. Class. Quantum Grav. 23: 4353.

6. Sharma, S.K. and Khanal, U. 2014. Perturbation of FRW Spacetime In NP Formalism. International Journal of Modern Physics D. 23(2): 1450017.

7. Chandrasekhar, S. 1983. The Mathematical Theory of Black Holes Clarendon Press, Oxford.

8. Abramowitz, M. and Stegun, I. A. 1976. Handbook of Mathematical Functions. Dover. New York.

9. Walker, M. 1970. Mathematical Methods of Physics. Pearson Education. p. 279. 